\title{
Ligand-Functionalized Poly(ethylene glycol) Particles for Tumor Targeting and Intracellular Uptake
}

Jiwei Cui ${ }^{\dagger,+, \perp}$ Karen Alt,${ }^{\S, \perp}$ Yi Ju, ${ }^{+, \perp}$ Sylvia T. Gunawan,,+ Julia A. Braunger,,+ Ting-Yi Wang,,+

Yunlu Dai, ${ }^{\ddagger}$ Qiong Dai, ${ }^{\dagger+}$ Joseph J. Richardson, ${ }^{+}$Junling Guo, ${ }^{+}$Mattias Björnmalm,,+

Christoph E. Hagemeyer, ${ }^{*}$, and Frank Caruso $*, *$

$\dagger$ Key Laboratory of Colloid and Interface Chemistry of the Ministry of Education, School of Chemistry and Chemical Engineering, Shandong University, Jinan, Shandong 250100, China

† ARC Centre of Excellence in Convergent Bio-Nano Science and Technology, and the

Department of Chemical and Biomolecular Engineering, The University of Melbourne, Parkville, Victoria 3010, Australia

$\S$ Nanobiotechnology Laboratory, Australian Centre for Blood Diseases, Monash University, Melbourne, Victoria 3004, Australia

KEYWORDS: mesoporous silica particles, PEG particles, RGD, intracellular uptake, targeting

ABSTRACT: Drug carriers typically require both stealth and targeting properties to minimize nonspecific interactions with healthy cells and increase specific interaction with diseased cells. Herein, the assembly of targeted poly(ethylene glycol) (PEG) particles functionalized with 
cyclic peptides containing Arg-Gly-Asp (RGD) (ligand) using a mesoporous silica templating method is reported. The influence of PEG molecular weight, ligand-to-PEG molecule ratio, and particle size on cancer cell targeting to balance stealth and targeting of the engineered PEG particles is investigated. RGD-functionalized PEG particles (PEG-RGD particles) efficiently target U-87 MG cancer cells under static and flow conditions in vitro, whereas PEG and cyclic peptides containing Arg-Asp-Gly (RDG)-functionalized PEG (PEG-RDG) particles display negligible interaction with the same cells. Increasing the ligand-to-PEG molecule ratio improves cell targeting. In addition, the targeted PEG-RGD particles improve cell uptake via receptor-mediated endocytosis, which is desirable for intracellular drug delivery. The PEGRGD particles show improved tumor targeting (14\% ID g $^{-1}$ ) when compared with the PEG (3\% $\mathrm{ID}^{-1}$ ) and PEG-RDG (7\% $\mathrm{ID} \mathrm{g}^{-1}$ ) particles in vivo, although the PEG-RGD particles show comparatively higher spleen and liver accumulation. The targeted PEG particles represent a platform for developing particles aimed at balancing nonspecific and specific interactions in biological systems.

\section{INTRODUCTION}

Nonspecific biological interactions of drug carriers are considered to be significant barriers to affording substantial accumulation of the therapeutic drug at diseased sites, therefore limiting the efficacy of drug delivery. ${ }^{1-3}$ To avoid nonspecific interactions, surface modification with poly(ethylene glycol) (PEGylation) is a widely used strategy, as it can endow drug carriers with improved biodistribution and pharmacokinetic profiles. ${ }^{4,5}$ However, the antibiofouling (stealth) 
property of PEGylation is governed by the molecular weight, grafting density, and conformation of PEG molecules on the carrier surface, all of which can be difficult to control and finely tune. ${ }^{6-8}$ To circumvent the challenges associated with PEGylation, we previously reported particles composed of PEG, engineered using a mesoporous silica (MS) templating method. ${ }^{9}$ These PEG particles showed substantially improved biodistribution and circulation behavior after optimization of the particle size and PEG molecular weight. In addition, the Young's modulus (or stiffness) of these PEG particles could be controlled from 0.2 to $3.3 \mathrm{kPa}$ by changing the cross-linking density, and their deformability could be tuned to be comparable to that of red blood cells ( $26 \mathrm{kPa})$, as demonstrated using a microfluidic capillary model. ${ }^{10}$ This enhanced deformability of the PEG particles can potentially enhance retention by bypassing biological barriers in vivo. Recent studies have also demonstrated that PEG-based particles can be used for chemotherapy and multimodel-imaging-guided combination therapy. ${ }^{11-13}$ Despite the significant advances achieved to date in passively targeted particles for cancer therapy, achieving a balance between low nonspecific interactions and high active targeting remains a limiting factor in the translation of safer and more efficient nanomedicines, and needs to be carefully considered in the design of drug delivery systems. ${ }^{14,15}$

The use of PEG can help protect drug carriers from mononuclear phagocytic system clearance and can thereby increase the circulation time and tumor accumulation (e.g., in tumors exhibiting the enhanced permeability and retention (EPR) effect). However, PEG can also hinder tumor cell uptake and intracellular drug release, which is not desirable for therapeutic delivery. ${ }^{16-18}$ An approach to overcome this is via the attachment of targeting molecules to PEG-based particles to increase specific interactions and induce receptor-mediated uptake. ${ }^{19}$ 
Synergistic integration of stealth and targeting properties by tuning the number of targeting ligands to confer maximum targeting performance, while maintaining stealth performance, offers a promising pathway to design particles with high drug delivery efficacy. ${ }^{20,21}$

Recently, we have functionalized PEG particles with bispecific antibodies (BsAbs) to modulate targeting of stealth PEG particles to tumor cells. ${ }^{22}$ However, the addition of targeting molecules often changes the surface chemistry of the particles and can possibly negatively influence the stealth properties of the particles. That is, nonspecific (stealth) and specific (targeting) interactions can compromise each other. For example, higher densities of targeting ligands (e.g., folic acid and aptamers) on PEGylated particle surfaces typically induce faster clearance or increased nonspecific accumulation (e.g., liver or spleen uptake). ${ }^{15,23}$ Although in our previous study PEG-BsAb particles displayed high association with cancer cells in vitro while retaining low nonspecific interaction with immune cells (i.e., monocytes and granulocytes) ex vivo, these particles did not display significantly improved tumor targeting in vivo. ${ }^{22}$ In addition, the length of the PEG molecules should be shorter than the length of the ligand linker to avoid interference with ligand-receptor interactions. ${ }^{24,25}$

Cyclic peptides containing Arg-Gly-Asp-Phe-Lys (cRGDfK, abbreviated as RGD) sequence can be recognized by integrins (e.g., the $\alpha_{v} \beta_{3}$ segment) overexpressed at angiogenic sites and in many tumors and have been widely used as targeting ligands for drug delivery ${ }^{26}$ and bioimaging, ${ }^{27}$ and in clinical trials. ${ }^{28,29}$ Although the RGD density on PEGylated vesicles or silica nanoparticles influences their circulation time and targeting outcomes, ${ }^{30,31}$ a detailed investigation on balancing stealth and active targeting of PEG particles has not been reported 
to date. Herein, we report the preparation of targeted PEG particles and examine the effect of PEG molecular weight, ligand-to-PEG molecule ratio, and particle size on cancer cell targeting and biodistribution (Scheme 1). 8-Arm-PEG-NH2 at two different molecular weights (10 or 40 $\mathrm{kDa})$ was used as a building block and silica particles with different sizes (110 or $1000 \mathrm{~nm})$ were used as templates for the synthesis of the PEG particles. RGD and control cyclic peptides of Arg-Asp-Gly-Phe-Lys (cRDGfK, abbreviated as RDG) were used to investigate: 1) the influence of surface functionalization of PEG particles on biodistribution; 2) the differences between active and passive tumor targeting; and 3) optimal strategies for controlling PEG particle bio-nano interactions (Scheme 1).

We investigated the bio-nano interactions of PEG particles using static cell culture, flow-based cell culture, and in vivo xenograft mice models and found important differences between the ex vivo and in vivo models. For the RGD-functionalized PEG (PEG-RGD) particles, increasing the molecular weight of PEG reduced specific targeting to integrin-overexpressing cancer cells (U-87 MG), but this could be improved by increasing the amount of RGD on the particles. The PEG-RGD particles efficiently targeted cancer cells (both under static and in flow conditions), whereas the (nonfunctionalized) PEG and RDG-functionalized PEG (PEG-RDG) particles had negligible interaction with the same cells. Furthermore, the targeted PEG particles showed improved cell uptake. In addition, PEG-RGD particles displayed higher tumor accumulation than the PEG and PEG-RDG particles in vivo, although the PEG-RGD particles displayed relatively higher spleen and liver accumulation. This result highlights that surface modification of PEG particles with RGD targeting ligands can be engineered to improve tumor targeting as 
well as enhanced cell uptake via receptor-mediated endocytosis, which is favorable for intracellular drug delivery (Scheme 1).

a

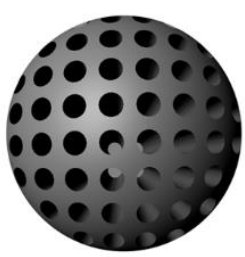
PEG loading and cross-linking

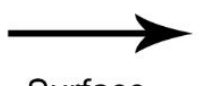

Surface

MS template functionalization
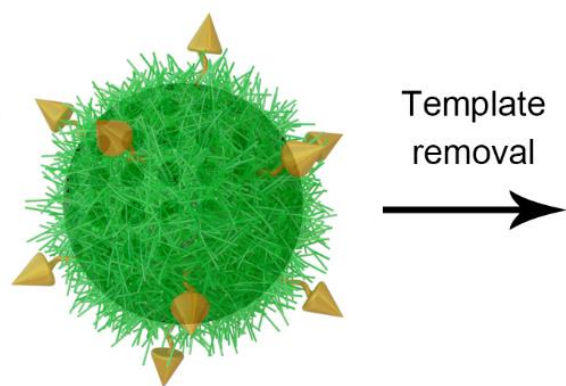

b
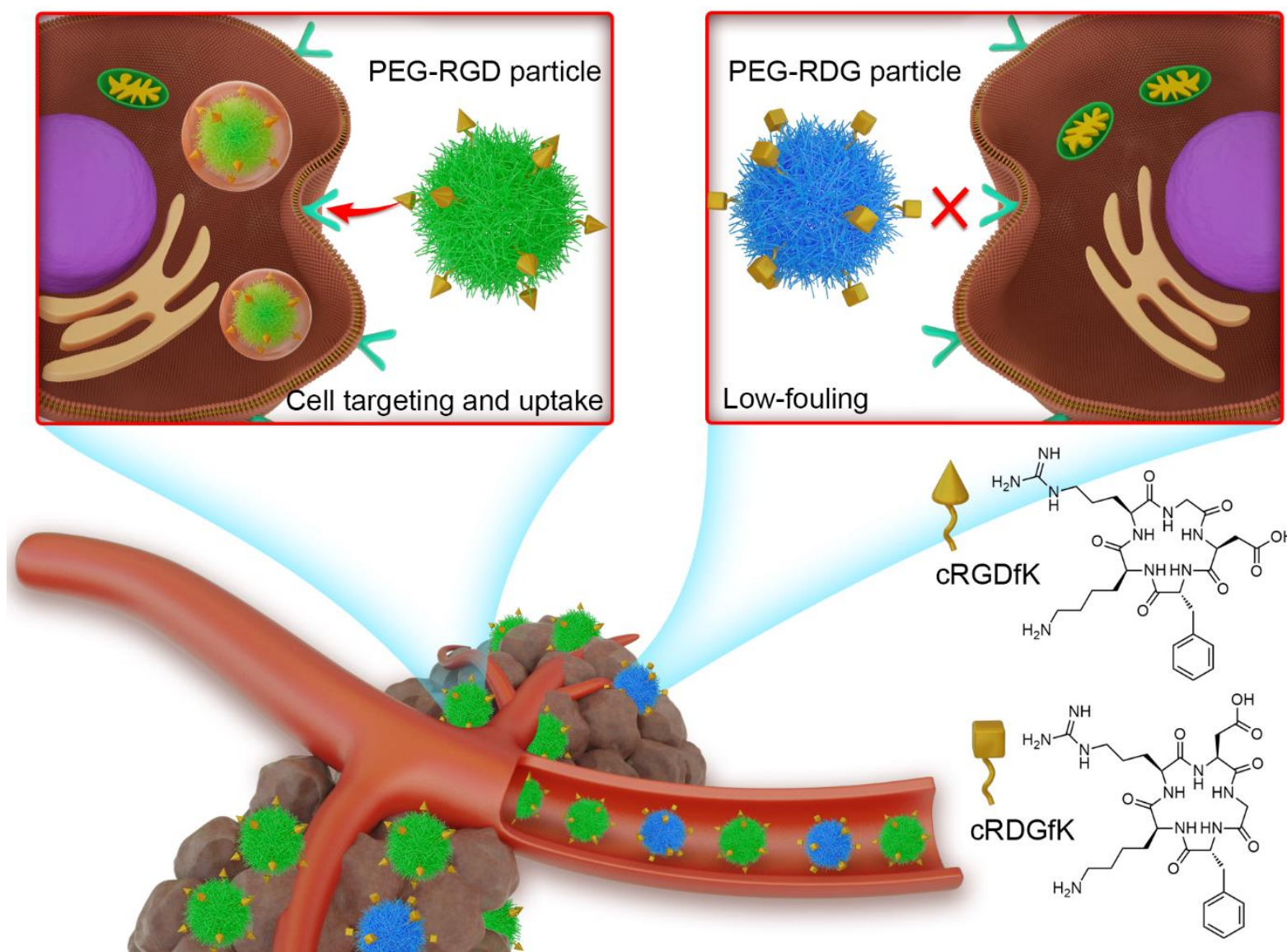

Scheme 1. (a) Schematic of the preparation of PEG-RGD particles via MS templating method.

(b) Illustrations of the tumor targeting and intracellular uptake of PEG-RGD particles in comparison to PEG-RDG particles. 
Materials. Tetraethyl orthosilicate (TEOS), poly(acrylic acid) (PAA, $M \mathrm{w} \sim 250 \mathrm{kDa}, 35 \mathrm{wt} \%$ solution in water), cetyltrimethylammonium bromide (CTAB), cetyltrimethylammonium tosylate (CTAT), triethanolamine, sodium phosphate dibasic, L-glutamine solution $(200 \mathrm{mM})$, Dulbecco's phosphate buffered saline (DPBS), hydrofluoric acid (HF, 48 wt\%), ammonium fluoride $\left(\mathrm{NH}_{4} \mathrm{~F}\right)$, ammonium hydroxide solution (28-30\%), anhydrous dimethyl sulfoxide (DMSO), and U-87 MG cell line were obtained from Sigma-Aldrich (Australia). 8-Arm-PEG$\mathrm{NH}_{2}$ (10 and $40 \mathrm{kDa}$ ) and 8-arm-PEG succinimidyl succinate (8-arm-PEG-NHS, $10 \mathrm{kDa}$ ) with a hexaglycerol core structure were purchased from JenKem Technology USA Inc. (China). Alexa Fluor 488 carboxylic acid succinimidyl ester (AF488), Alexa Fluor 647 carboxylic acid succinimidyl ester (AF647), Alexa Fluor 594-conjugated wheat germ agglutinin (WGAAF594), WGA-AF488, Hoechst 33342, heat-inactivated fetal bovine serum (HI-FBS), and advanced minimum essential medium (MEM) were provided by Invitrogen (Australia). Cyclic peptides containing Arg-Gly-Asp-Phe-Lys (cRGDfK), Arg-Asp-Gly-Phe-Lys (cRDGfK), and Arg-Gly-Asp-Sec-Lys (cRGDUK) were purchased from GL Biochem (Shanghai) Ltd. (China). The water used in all experiments was obtained from a three-stage Millipore Milli-Q Plus 185 purification system and had a resistivity greater than $18.2 \mathrm{M} \Omega \mathrm{cm}$.

Minimum Information Reporting in Bio-Nano Experimental Literature (MIRIBEL). The studies conducted herein, including material characterization, biological characterization, and experimental details, conform to the MIRIBEL reporting standard for bio-nano research, ${ }^{32}$ and we include a companion checklist of these components in the Supporting Information. 
Synthesis of MS Particles. For the synthesis of MS particles with an average size of $1000 \mathrm{~nm}$, polyelectrolyte-surfactant complexes were used as templates. ${ }^{33}$ Briefly, CTAB (1.1 g) was completely dissolved in water $(50 \mathrm{~mL})$ with stirring. PAA solution $(4.3 \mathrm{~g})$ was then added with vigorous stirring at room temperature $\left(25^{\circ} \mathrm{C}\right)$ to obtain a clear solution. Ammonium hydroxide solution $(3.5 \mathrm{~mL})$ was then added to the above solution with vigorous stirring for $20 \mathrm{~min}$, producing a milky suspension. TEOS $(4.46 \mathrm{~mL})$ was then added to the above solution. Following stirring for $15 \mathrm{~min}$, the mixture was transferred to a Teflon-sealed autoclave, which was heated at $100{ }^{\circ} \mathrm{C}$ for $48 \mathrm{~h}$. The resulting particles were washed with water and ethanol three times and dried at $80{ }^{\circ} \mathrm{C}$. The templates were removed by calcination at $550{ }^{\circ} \mathrm{C}$ in air for $6 \mathrm{~h}$. MS particles with an average size of $110 \mathrm{~nm}$ were synthesized according to a previously reported method. ${ }^{34}$ Briefly, CTAT $(960 \mathrm{mg})$ and triethanolamine $(174 \mathrm{mg})$ were dissolved in water $(50 \mathrm{~mL})$ at $80{ }^{\circ} \mathrm{C}$, followed by prompt addition of TEOS $(7.8 \mathrm{~mL})$. The mixture was vigorously stirred at $80{ }^{\circ} \mathrm{C}$ for another $2 \mathrm{~h}$. The synthesized MS particles were washed with water and ethanol, dried at $80{ }^{\circ} \mathrm{C}$, and calcined at $550{ }^{\circ} \mathrm{C}$ in air for $6 \mathrm{~h}$.

Synthesis of PEG, PEG-RDG, and PEG-RGD Particles. As per the previously published protocol, ${ }^{35} \mathrm{MS}$ particles ( 6 mg) were washed with phosphate buffer (100 mM, pH 8) and infiltrated with 8 -arm-PEG-NH 2 solution $\left(480 \mu \mathrm{L}, 5 \mathrm{mg} \mathrm{mL}^{-1}\right.$ in phosphate buffer) with constant shaking overnight. The particles were then isolated by centrifugation $(1500 \mathrm{~g}, 2 \mathrm{~min}$ for particles prepared using MS-1000 and $8000 \mathrm{~g}, 8 \mathrm{~min}$ for particles prepared using MS-110) and washed three times with phosphate buffer. The pellet was re-dispersed in phosphate buffer $(100 \mu \mathrm{L})$ and labeled with AF488 $\left(5 \mu \mathrm{L}, 1 \mathrm{mg} \mathrm{mL}^{-1}\right)$ or AF647 $\left(5 \mu \mathrm{L}, 1 \mathrm{mg} \mathrm{mL}^{-1}\right)$, followed by polymer cross-linking with 8 -arm-PEG-NHS $\left(20 \mathrm{mg} \mathrm{mL}^{-1}\right.$ in anhydrous DMSO, $\left.40 \mu \mathrm{L}\right)$ in 
phosphate buffer $(400 \mu \mathrm{L})$. After incubation for $2 \mathrm{~h}$, the particles were washed with water three times and resuspended in water $(200 \mu \mathrm{L})$. To functionalize the PEG particles with RDG or RGD, 8-arm-PEG-NHS (0.8 mg) was pre-incubated with RDG or RGD in anhydrous DMSO at a peptide/8-arm-PEG-NHS molar ratio of 1:1 or 2:1 in anhydrous DMSO for $30 \mathrm{~min}$. To obtain the PEG particles or PEG-RDG and PEG-RGD particles, the MS templates were removed with a $\mathrm{HF}(2 \mathrm{M}) / \mathrm{NH}_{4} \mathrm{~F}(8 \mathrm{M})$ solution $(\mathrm{pH} \sim 5)$. Caution! HF is highly toxic. Extreme care should be taken when handling HF solution and only small quantities should be prepared. The resultant PEG, PEG-RDG, and PEG-RGD particles were washed three times with water and resuspended in water. The particles are denoted as PEG $x-y$-RGD or PEG $x-y$-RDG, where $x$ and $y$ represent PEG molecular weight (10 or $40 \mathrm{kDa})$ and template size $(110$ or $1000 \mathrm{~nm})$, respectively. It should be noted that the cross-linking of particles is less efficient and the yield of PEG-RGD particles (especially for PEG40-110-RGD or PEG40-110-RDG particles) is lower when the RGD-to-8-arm-PEG-NHS ratio is higher than 2:1.

Quantification of RGD Per PEG-RGD Particles. To quantify the amount of RGD conjugated on the PEG particles, cRGDUK was conjugated following the same protocol as above. The cRGDUK was employed to introduce $\mathrm{Se}$ (from $\mathrm{Sec}, \mathrm{U}$ ) into the peptide for the quantification. The Se concentration in the particle suspension was determined by inductively coupled plasma mass spectrometry (NexION-2000, PerkinElmer, USA). The concentration of the PEG particles was determined by weighing the freeze-dried PEG particles. From these data, the amount of cRGDUK per mg of PEG particles was estimated as the total Se concentration divided by the PEG particle concentration. As a result, when the mixing ratio of peptide to 8- 
arm-PEG-NHS is 2:1, the cRGDUK modification ratios are 10 and $3.5 \mu \mathrm{g}$ of cRGDUK per mg of PEG40-1000-RGD2 and PEG40-110-RGD2 particles, respectively.

Cell Association of PEG, PEG-RDG, and PEG-RGD Particles. U-87 MG cells (American Type Culture Collection) were cultured in a complete media (90\% advanced MEM, $10 \%$ HIFBS, and supplemented with $1 \%$ L-glutamine) at $37{ }^{\circ} \mathrm{C}$ in a $5 \% \mathrm{CO}_{2}$-humidified atmosphere. U-87 MG cells with passage number 20-30 were used and all cells passed the mycoplasma test. To investigate cell association, U-87 MG cells were seeded in a 24 -well plate at a population of 70000 cells per well (500 $\mu \mathrm{L}$ of media) and incubated overnight. Fluorescently labelled PEG10-1000, PEG10-1000-RDG, PEG10-1000-RGD, PEG40-1000, PEG40-1000RDG2, or PEG40-1000-RGD2 particles were added at a particle-to-cell ratio of 1000:1 to each well (23 $\mu \mathrm{g}$ of particles per mL of media). For PEG40-110, PEG40-110-RGD2, and PEG40110-RDG2 particles, $3.4 \mu \mathrm{g}$ of particles per mL of media was used for incubation with cells (this amount ensures optimal cell association). At different time points, the cells were gently washed with DPBS and detached with trypsin. The cell suspensions were centrifuged $(300 \mathrm{~g}$, 2 min) and washed with DPBS. Finally, the cells were suspended in of DPBS (500 $\mu$ L) and analyzed by flow cytometry (Apogee Micro Flow cytometer). The gating strategy is shown in Figure S7. Experiments were performed in triplicate.

Cell Imaging. U-87 MG cells were seeded at a density of 20000 cells per well (150 $\mu \mathrm{L}$ media) in 8-well Lab-Tek chambered coverglass slides (Thermo Fisher Scientific) and allowed to adhere overnight. Subsequently, cells were incubated with the PEG10-1000, PEG10-1000RDG2, PEG10-1000-RGD2，PEG40-1000, PEG40-1000-RDG2，or PEG40-1000-RGD2 
particles at a particle-to-cell ratio of $1000: 1$ for $24 \mathrm{~h}\left(37{ }^{\circ} \mathrm{C}, 5 \% \mathrm{CO}_{2}\right)$, followed by three washing steps with DPBS. For PEG40-110, PEG40-110-RDG2, and PEG40-110-RGD2 particles, $8.5 \mu \mathrm{g}$ of particles per $\mathrm{mL}$ of media was used for incubation with cells. Cells were fixed with of $4 \%$ paraformaldehyde $(200 \mu \mathrm{L})$ at $25^{\circ} \mathrm{C}$ for $15 \mathrm{~min}$, followed by three washing steps with DPBS. The fixed cells were stained with WGA-AF594 $\left(5 \mu \mathrm{g} \mathrm{mL} \mathrm{m}^{-1}\right.$ in DPBS) on ice for $10 \mathrm{~min}$ and Hoechst $33342\left(2 \mu \mathrm{g} \mathrm{mL} \mathrm{m}^{-1}\right.$ in DPBS $)$ for $15 \mathrm{~min}$ and washed with DPBS twice. Fluorescence microscopy imaging of cells was performed on a Nikon A1R confocal microscope equipped with a $60 \times$ oil immersion objective and a standard fluorescein isothiocyanate (FITC)/tetramethylrhodamine (TRITC)/cyanine 5 (CY5) filter set.

For multicellular spheroid imaging, U-87 MG cells were seeded in Corning spheroid microplates (96-well plate) at a density of 1000 per well and cultured for 4 days. The medium was replaced with fresh medium $(100 \mu \mathrm{L})$ containing PEG40-110, PEG40-110-RDG2, or PEG40-110-RGD2 particles ( $8.5 \mu \mathrm{g}$ of PEG particles per mL of media) labeled with AF647, and the incubation proceeded for $24 \mathrm{~h}$. The spheroids were washed with DPBS three times and fixed by $4 \%$ paraformaldehyde in DPBS at $25{ }^{\circ} \mathrm{C}$ for $15 \mathrm{~min}$, followed by cell membrane staining with WGA-AF488 (5 $\mu \mathrm{g} \mathrm{mL}^{-1}$ in DPBS) on ice for $10 \mathrm{~min}$ and washing with DPBS. To monitor particle association and penetration of multicellular spheroids (MCSs), imaging was performed on a Nikon A1R confocal microscope with a $20 \times$ objective and a standard FITC/CY5 filter set.

Microfluidic Assay. For flow live cell imaging, U-87 MG cells $\left(5 \times 10^{4}\right.$ cells per channel) were seeded into sterile ibidiTreat $\mu$-Slide $\mathrm{I}^{0.4}$ Luer flow chambers in advanced MEM medium 
supplemented with $10 \%$ HI-FBS, $1 \%$ glutamine, and $1 \%$ penicillin-streptomycin according to the manufacturer's instructions. The cells were cultured overnight at $37{ }^{\circ} \mathrm{C}$ with $5 \% \mathrm{CO}_{2}$. The flow experiments were performed using a setup consisting of a $50 \mathrm{~mL}$ centrifugation tube as a reservoir, a flow chamber (ibidiTreat $\mu$-Slide $I^{0.4}$ Luer), and a syringe pump (PHD2000, Harvard Apparatus) connected via silicone tubing (internal diameter (ID) $0.76 \mathrm{~mm}$, ibidi) and Luer connectors (Figure S4). The tubing and connectors were autoclaved before usage. The flow system was assembled under sterile conditions (in a biosafety cabinet). The reservoir was filled with prewarmed $\left(37^{\circ} \mathrm{C}\right)$ complete medium containing PEG40-1000-RGD2 or PEG401000-RDG2 particles labeled with AF647 at a concentration of $1.4 \times 10^{8}$ particles per $\mathrm{mL}$ of media, corresponding to the particle concentration used in the static cell association experiments. Before setting up the flow system, the inlet and outlet of the tubing were flushed with prewarmed medium and connected to the cell-containing flow chamber in a drop-to-drop fashion to avoid introduction of air bubbles. The assembled system was transferred onto a Nikon AR1 confocal microscope fitted with an incubator and connected to the syringe pump in withdraw mode for the flow experiments at a shear stress of $0.1 \mathrm{dyn} \mathrm{cm}^{-2}\left(\sim 110 \mu \mathrm{L} \mathrm{min}{ }^{-1}\right)$ at $37{ }^{\circ} \mathrm{C}$ for live cell imaging. The reservoir was kept under a stream of $5 \% \mathrm{CO}_{2}$ to maintain the required $\mathrm{CO}_{2}$ content for cell viability. Cell images were taken under the flow condition.

Tumor Inoculation. All procedures were followed in accordance with the Australian National Health and Medical Research Council's published Code of Practice for the Use of Animals in Research, and experiments were approved by the Alfred Medical Research and Education Precinct Animal Ethics Committee (E/1589/2015/B). Mice were housed on a $12 \mathrm{~h}$ light/dark cycle with ad libitum access to food and water. 
U-87 cells $\left(2 \times 10^{6}\right.$ in $100 \mu \mathrm{L}$ Matrigel and $100 \mu \mathrm{L}$ PBS $)$ were used to inoculate a subcutaneous tumor on the right shoulder of each male nude mouse (6 weeks old). The body weight and tumor volume were monitored twice a week. The tumor diameter $(D)$ was measured, and the corresponding tumor volume was calculated using the following formula: volume = $(4 / 3) \pi(D / 2)^{3}$. The xenograft tumor-bearing mice were randomized into three groups (PEG40110-RGD2, PEG40-110-RDG2, and PEG40-110) with $n=3$ per group.

In Vivo Fluorescence Imaging. When the tumor volume was $60 \mathrm{~mm}^{3}$, the mice were injected intravenously with PEG particles $(\sim 75 \mu \mathrm{g}$ of PEG particles in $100 \mu \mathrm{L}$ DPBS; particle suspensions have similar fluorescence intensities). The mice were anesthetized by isoflurane during the entire imaging procedure. The entire body of the mouse was imaged using an IVIS Lumina II Imaging System (Caliper Life Sciences) at 24 and $48 \mathrm{~h}$ post injection. The excitation and emission filters were 620 and $670 \mathrm{~nm}$, respectively. The total radiant efficiency $(\mathrm{p} / \mathrm{s}) /\left(\mu \mathrm{W} / \mathrm{cm}^{2}\right)$ is shown. All imaging parameters were kept constant and were as follows: Bin, (M)4; field of view (FOV), 12.5; f2; $2 \mathrm{~s}$.

Ex Vivo Fluorescence Imaging. After the last scan at $48 \mathrm{~h}$ post injection, the mice were euthanized by ketamine/xylazine overdose. The heart, liver, spleen, kidney, lung, and tumor were collected and weighed. The main organs were placed on a petri dish and imaged using an IVIS Lumina II imaging system (Caliper Life Sciences). All imaging parameters were kept constant and were as follows: Bin, (M)4; FOV, 12.5; f2; 2 s. The total radiant efficiency $(\mathrm{p} / \mathrm{s}) /\left(\mu \mathrm{W} / \mathrm{cm}^{2}\right)$ of the organs as well as the standards (injected samples) were measured and 
background corrected. Based on the weight of the individual organs, this was then converted to $\% \mathrm{ID} \mathrm{g}^{-1}$.

Tissue Processing, Immunohistochemistry, and Imaging. The tumors were collected and post-fixed for $2 \mathrm{~h}$ in $4 \%$ paraformaldehyde and cryo-protected for 3 days in sucrose $(30 \% \mathrm{w} / \mathrm{v}$ in $0.1 \mathrm{M}$ phosphate buffered saline) before being coronally sectioned on a cryostat (12 series collected at a section thickness of $40 \mu \mathrm{m}$ ). The tissue was mounted on slides and air dried for $1 \mathrm{~h}$ prior to storage at $-80{ }^{\circ} \mathrm{C}$. Tumor tissues were stained with plasma membrane marker WGA-AF488 (1:1000, Thermo Fisher Scientific) and nuclear marker Hoechst 33342 (1:1000, Invitrogen), followed by three washes with DPBS. Fluorescence images were captured using a Nikon A1R confocal microscopy with a 40x water immersion objective.

Characterization Methods. Gas sorption analysis, using nitrogen as the adsorption gas, was performed on a Micromeritics Tristar surface area and porosity analyzer to determine the porosity and surface area of the MS particles. The pore size distributions were derived from the desorption curve using the Barrett-Joyner-Halenda method. Transmission electron microscopy (TEM) analysis was carried out on a FEI Tecnai TF20 microscope operating at 200 $\mathrm{kV}$. Fluorescence microscopy images of the particles were taken using an Olympus IX71 inverted fluorescence microscope equipped with a differential interference contrast slider (UDICT, Olympus), the corresponding filter sets, and a $60 \times$ oil immersion objective (Olympus UPFL20/0.5NA, W.D. 1.6). Particle counting and the cell association assay were performed on an Apogee Micro Flow Cytometer at an excitation wavelength of 488 or $647 \mathrm{~nm}$. Confocal 
laser scanning microscopy (CLSM) was performed on a Nikon A1R confocal microscope with a standard FITC/TRITC/CY5 filter set.

\section{RESULTS AND DISCUSSION}

Fabrication of Targeted PEG Particles. To prepare the peptide-functionalized PEG particles, monodisperse MS particles of average sizes of 1000 and $110 \mathrm{~nm}$ (denoted MS-1000 and MS110, respectively) were synthesized, as previously reported r $^{33,34}$ (Figure 1a,b). The MS-1000 particles displayed a bimodal pore size distribution centered at 2-4 and 10-40 nm, whereas the MS-110 particles featured pore sizes of 5-15 $\mathrm{nm}$. The surface areas of the MS-1000 and MS110 particles were 550 and $300 \mathrm{~m}^{2} \mathrm{~g}^{-1}$, respectively. The MS template particles were infiltrated with 8-arm-PEG-NH 2 , and cross-linking proceeded using 8-arm-PEG-NHS, which was prefunctionalized with RGD or RDG at different ratios in anhydrous DMSO (peptide/8-arm-PEGNHS ratio of 1:1 or 2:1). The template was then removed to obtain the peptide-functionalized PEG particles. Pre-functionalization of the peptide resulted in 0.8 and 1.4 peptides per 8 -armPEG-NHS when the ratios of the peptide/8-arm-PEG-NHS were 1:1 and 2:1, respectively, based on NMR analysis (Figure S1). The molecular structures and cross-linking strategy are shown in Figure S2. Previously, we have reported that PEG particles composed of higher molecular weight PEG (e.g., $40 \mathrm{kDa}$ ) displayed lower levels of cell association with phagocytic blood cells (i.e., monocytes and granulocytes). ${ }^{9}$ Herein, we used two different molecular weights (10 or $40 \mathrm{kDa}$ ) of 8-arm-PEG-NH 2 (each arm 1.25 or $5 \mathrm{kDa}$ ) for the synthesis of RGDfunctionalized PEG particles to investigate the balance between stealth and targeting performance. Figure 1c,d shows TEM images of PEG10-1000-RGD and PEG40-1000-RGD 
particles, which are similar in morphology to the PEG particles that we previously reported. ${ }^{9}$ The contrast of the PEG10-1000-RGD particles appears different from that of the PEG401000-RGD particles, which is likely due to the higher loading amount of 8-arm-PEG-NH $2(10$ $\mathrm{kDa})$ in MS-110 templates and the higher cross-linking density of the PEG10-1000-RGD particles after template removal. Fluorescence microscopy images showed that both the PEG40-1000-RGD and PEG40-110-RGD particles were well dispersed in aqueous solution (Figure 1e,f).
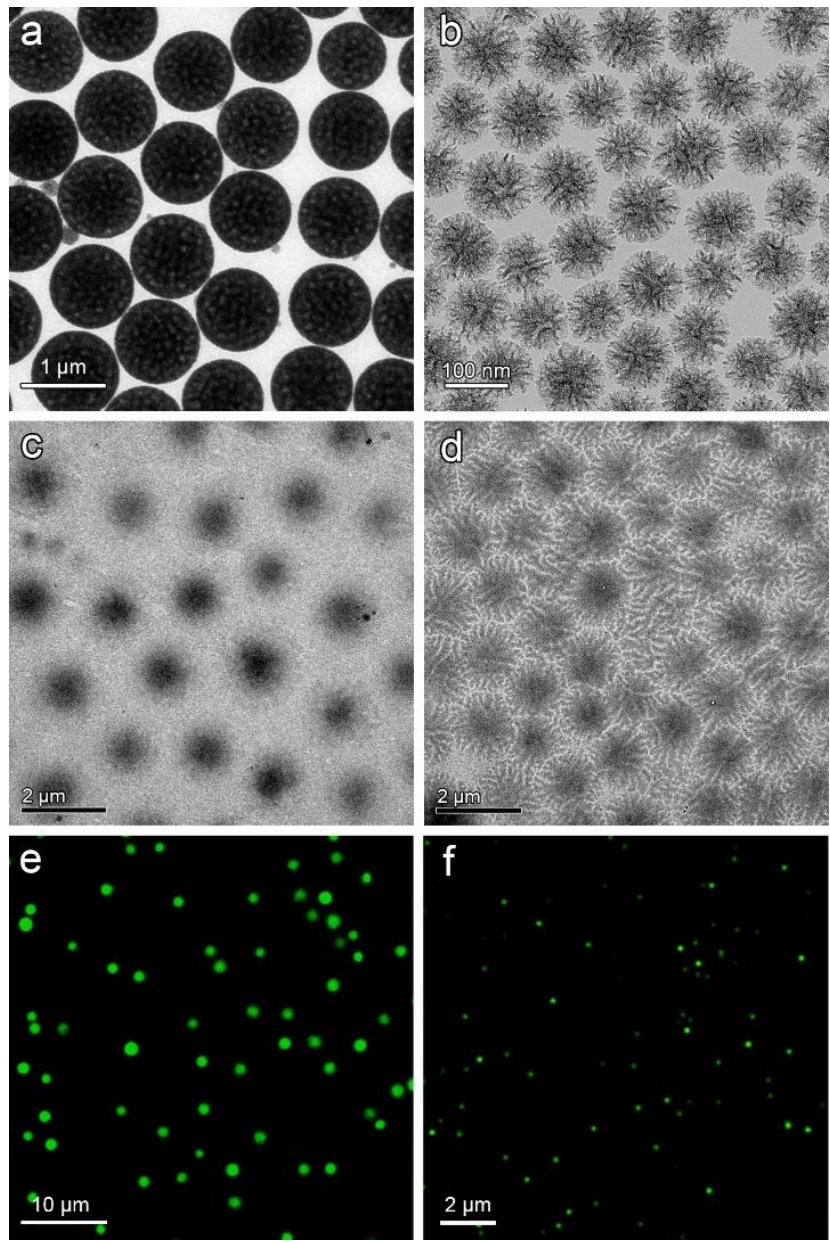

Figure 1. TEM images of (a) MS-1000 and (b) MS-110 templates, and (c) PEG10-1000-RGD and (d) PEG40-1000-RGD particles. (e) Fluorescence microscopy image of PEG40-1000-RGD 
particles. (f) Structured illumination microscopy image of PEG40-110-RGD particles. Particles were labeled with AF488.

In Vitro Cell Targeting and Internalization. The cyclic peptide containing an RGD sequence is a targeting moiety that can be recognized by $\alpha_{v} \beta_{3}$ integrin segment overexpressed on U-87 MG cells. ${ }^{36}$ To investigate cell targeting of the PEG-RGD particles, U-87 MG cells were incubated with PEG10-1000-RGD and PEG10-1000-RDG particles separately with a particleto-cell ratio of 1000:1 (23 $\mu \mathrm{g}$ of particles per mL of media). About $70 \%$ of U-87 MG cells associated with PEG10-1000-RGD particles after incubation for $24 \mathrm{~h}$, as monitored by flow cytometry, indicating efficient targeting of cancer cells. In contrast, the PEG10-1000-RDG particles showed nonspecific interactions, resulting in $\sim 25 \%$ of cell association after incubation for $24 \mathrm{~h}$ (Figure 2a). In an attempt to further decrease the nonspecific interactions, a higher PEG molecular weight was used. Figure $2 b$ shows that the nonspecific interaction of PEG401000-RDG particles was lower, with cell association decreasing to $\sim 5 \%$, whereas the specific targeting of PEG40-1000-RGD particles decreased to $15 \%$. This is consistent with our previous finding that using 8-arm PEG with a higher molecular weight as building block leads to higher resistance of the PEG particles to cellular interactions. ${ }^{9}$ Modulation of the targeting specificity of the PEG-RGD particles was then examined by tuning the peptide/8-arm-PEGNHS ratio. When increasing the peptide/8-arm-PEG-NHS ratio to 2:1, cell association of the resulting particles (denoted as PEG40-1000-RGD2) improved ( 30\%) (Figure 2b). In contrast, negligible cell association of PEG40-1000-RDG2 particles was observed (Figure 2b). Therefore, increasing the ratio of peptide to 8-arm-PEG-NHS did not negatively influence the stealth performance of the PEG particles in vitro, while still allowing for enhanced targeting. 
Moreover, the RDG-functionalized PEG particles behaved similarly to the PEG particles, further confirming that the peptides did not reduce the stealth performance of the PEG particles in vitro. The maximum projection of CLSM images confirmed the above flow cytometry results. Fewer PEG10-1000 or PEG10-1000-RDG2 particles associated with U-87 MG cells, whereas more PEG10-1000-RGD2 particles (average three particles per cell) associated with U-87 MG cells (Figure 2c). PEG40-1000-RGD2 particles also associated with U-87 MG cells, whereas cell association was not observed for PEG40-1000 or PEG40-1000-RDG2 particles (Figure 2d). To examine the cell internalization of the PEG particles, cross-sectional CLSM images were analyzed (Figure S3). The PEG-RGD particles, which were labeled with AF488 (green dots), were surrounded by the membrane (red) of the U-87 MG cells, demonstrating internalization of the PEG-RGD particles after cell targeting. 

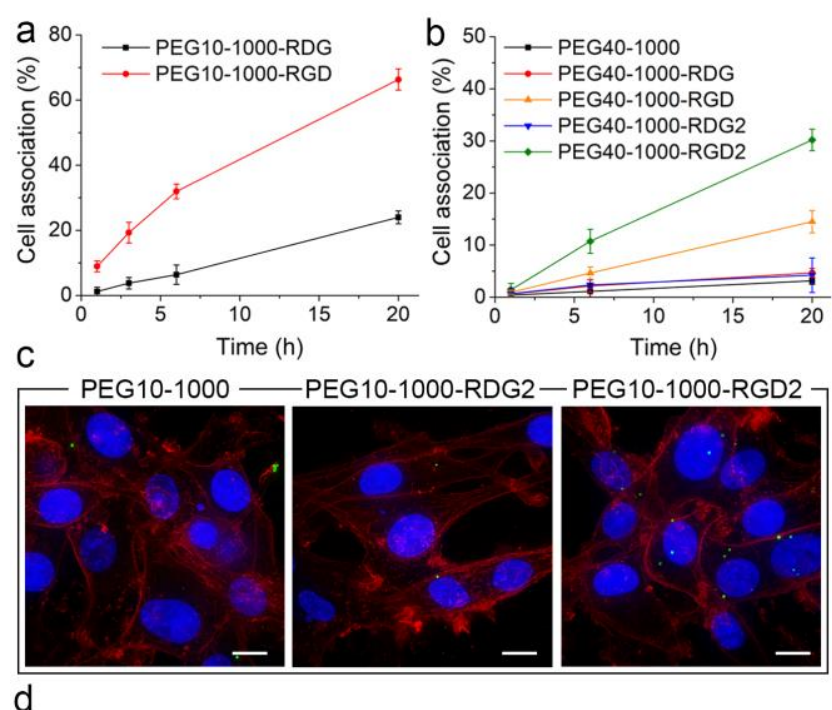

PEG10-1000-RDG2 - PEG10-1000-RGD2
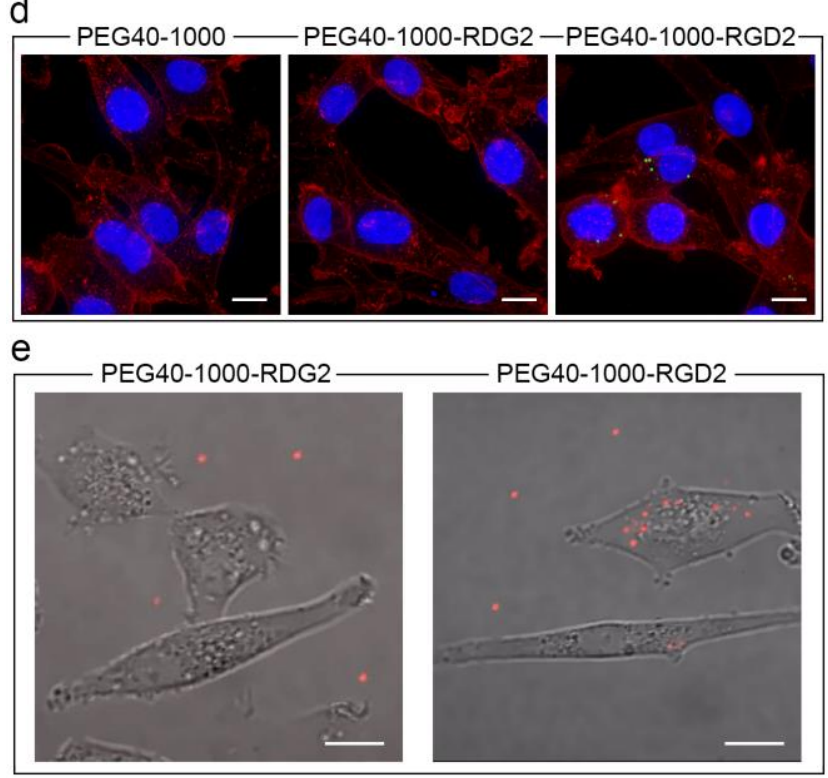

Figure 2. (a) Cell association of PEG10-1000-RGD and PEG10-1000-RDG particles. (b) Cell association of PEG40-1000, PEG40-1000-RDG, PEG40-1000-RGD, PEG40-1000-RDG2, and PEG40-1000-RGD2 particles. Data represent means \pm standard deviations based on triplicate experiments. (c,d) CLSM images (maximum projection) of cell association of PEG particles with and without ligand functionalization. Particles (green) were labeled with AF488. Cell membranes (red) and nuclei (blue) were stained with WGA-AF594 and Hoechst 33342, respectively. Scale bars are $10 \mu \mathrm{m}$. (e) CLSM images (maximum projection) showing cell 
association of PEG40-1000-RDG2 and PEG40-1000-RGD2 particles after incubation for 45 min under flow. Particles (red) were labeled with AF647. Scale bars are $10 \mu \mathrm{m}$.

Typically, to study targeted drug delivery in vivo, the drug carriers are intravenously injected and circulated under flow in the bloodstream. Microfluidic devices have been used to mimic in vivo microenvironments to study the behavior of nanoengineered particles under flow. ${ }^{37-41}$ To investigate the cell targeting of RGD-functionalized PEG particles under flow, we designed a microcirculation system consisting of a reservoir for cell media, a flow chamber where the cells are precultured, and a syringe pump connected via silicone tubing (Figure S4). PEG40-1000RGD2 or PEG40-1000-RDG2 particles at a concentration of $1.4 \times 10^{8}$ particles $\mathrm{mL}^{-1}$ (of media) (equivalent concentration to that used in the static cell culture condition) were examined in the microfluidic experiments. As observed by CLSM, the PEG40-1000-RGD2 particles targeted U-87 MG cells effectively under flow, whereas the PEG40-1000-RDG2 particles were stealthy under flow conditions (Figures 2e, S4 and Movies S1, S2), as consistent with the results from the static cell culture assay. 

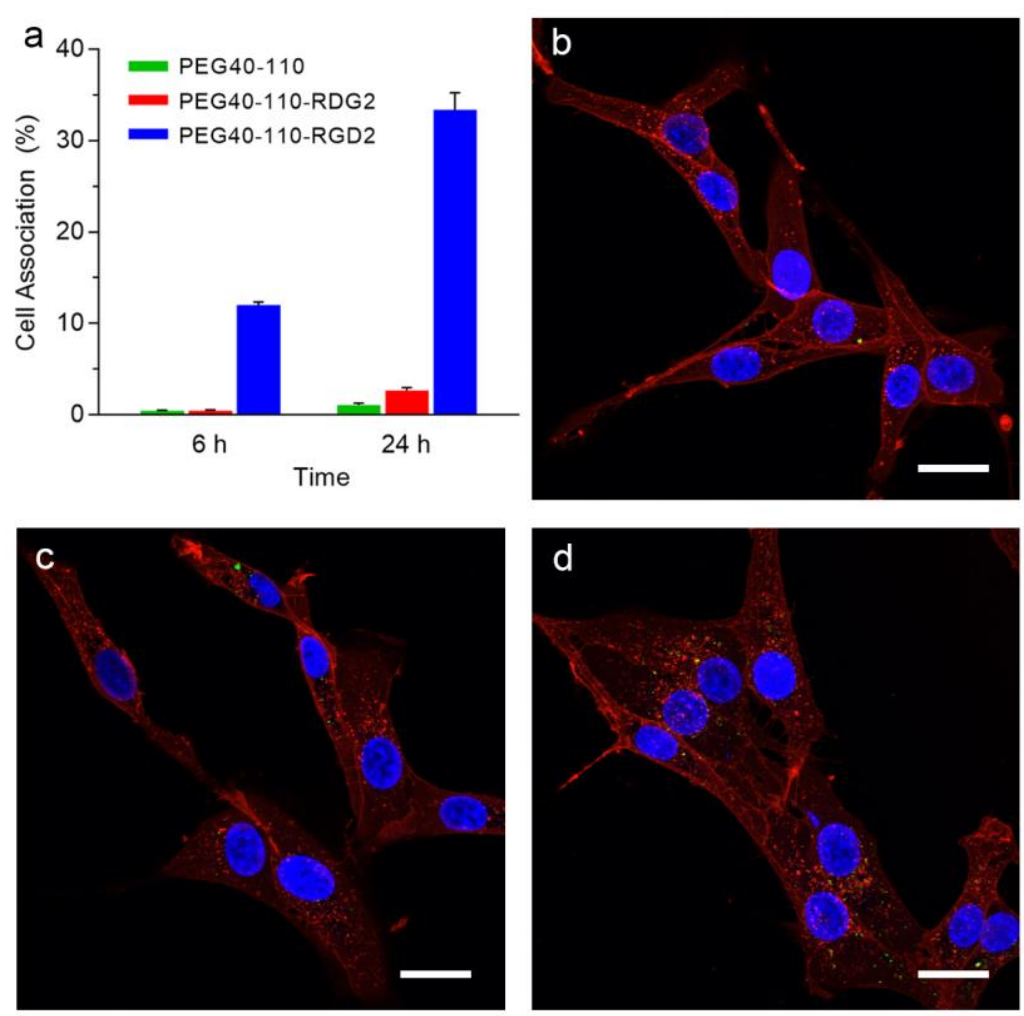

Figure 3. (a) Cell association of PEG40-110, PEG40-110-RDG2, and PEG40-110-RGD2 particles. Data represent means \pm standard deviations based on triplicate experiments. CLSM images (maximum projection) of cell association of (b) PEG40-110, (c) PEG40-110-RDG2, and (d) PEG40-110-RGD2 particles. Particles (green) were labeled with AF488. Cell membranes (red) and nuclei (blue) were stained with WGA-AF594 and Hoechst 33342, respectively. Scale bars are $10 \mu \mathrm{m}$.

According to the above experiments, using a higher molecular weight of PEG (i.e., $40 \mathrm{kDa}$ ) and a higher ligand/8-arm-PEG-NHS ratio (i.e., 2:1) can balance stealth and cell targeting to simultaneously avoid nonspecific interactions and improve specific targeting in vitro. Therefore, PEG40-110-RGD2 and PEG40-110-RDG2 particles were used for investigating cell targeting in vitro and in vivo. Both PEG40-110 and PEG40-110-RDG2 particles displayed negligible cell association (Figure 3a), similarly to PEG40-1000-RDG2. In contrast, PEG40- 
110-RGD2 particles displayed 12\% and 33\% cell association after incubation for 6 and $24 \mathrm{~h}$, respectively. These results indicate that RGD modification assists with targeting PEG particles to cancer cells regardless of particle size. Moreover, fewer PEG40-110-RGD2 particles (seven times less) were required to achieve the same cancer cell targeting performance as that achieved with PEG40-1000-RGD2 particles, thus highlighting the importance of small particle size for the improvement of cell targeting. In addition, CLSM images confirmed that significantly more PEG40-110-RGD2 particles could target cancer cells when compared with PEG40-110 and PEG40-110-RDG2 particles (Figure 3b-d).

In Vivo Tumor Biodistribution. MCSs have been introduced as a versatile 3D model for tumor biology studies. ${ }^{42}$ To investigate tumor targeting and penetration in vitro, an MCS model derived from U-87 MG cells was chosen. As shown in Figure 4a, the MCSs have a spherical structure with an average diameter of $450 \mu \mathrm{m}$. After incubation with PEG40-110, PEG40-110RDG2, or PEG40-110-RGD2 particles, the MCSs were imaged using CLSM. Cell association was only observed for the PEG40-110-RGD2 particles (Figure 4a). Importantly, more than 90\% of the associated particles was internalized by the U-87 MG cells, according to cross-sectional analysis of the CLSM images (Figure S5). The high level of internalization is likely due to RGD-specific $\alpha_{\mathrm{v}} \beta_{3}$ integrin-mediated endocytosis. 


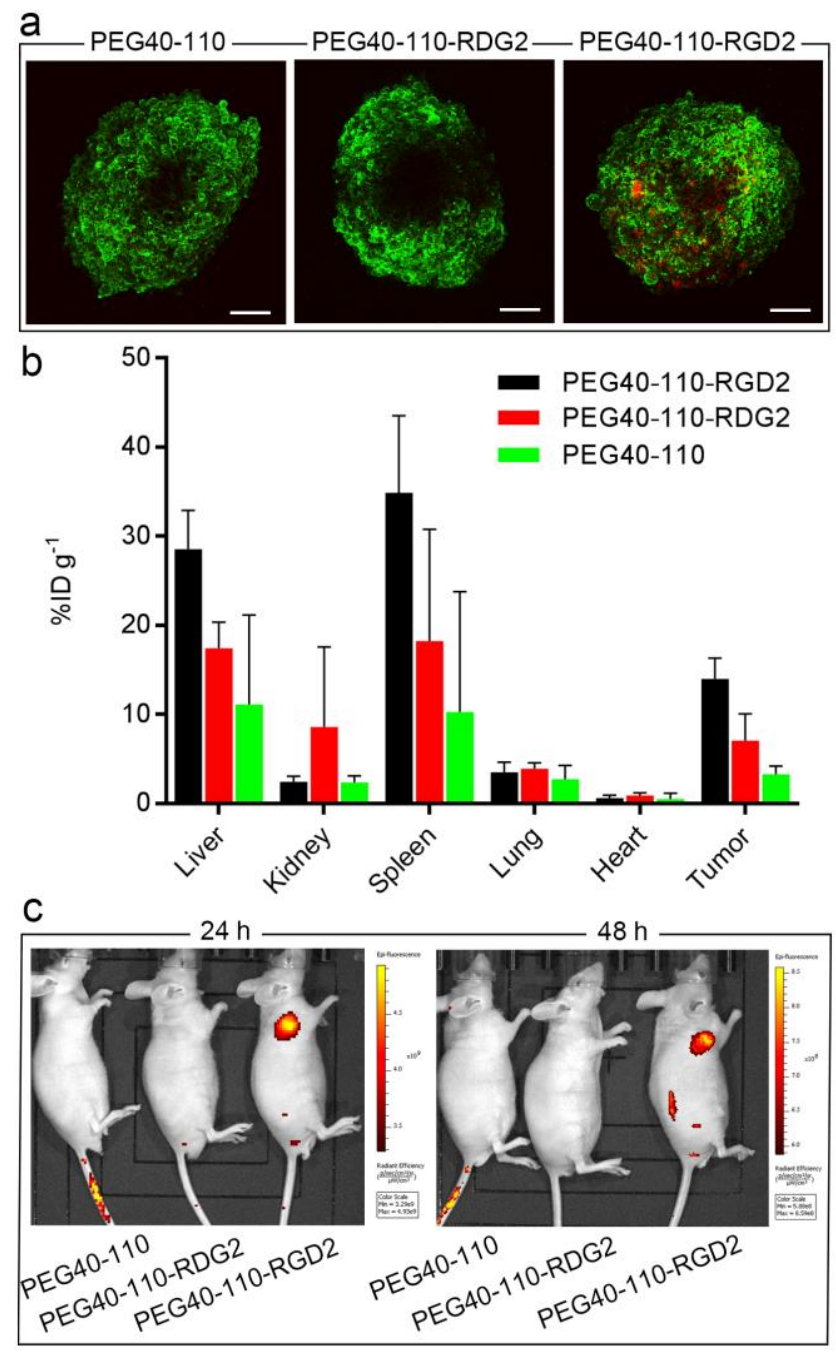

Figure 4. (a) Multicellular spheroid association and (b) biodistribution of PEG40-110, PEG40110-RDG2, and PEG40-110-RGD2 particles. Cell membranes (green) were stained with WGA-AF488. Particles (red) were labeled with AF647. Scale bars are $100 \mu \mathrm{m}$. The biodistribution of the different PEG particles in various organs is shown at $48 \mathrm{~h}$ after intravenous injection. Data represent means \pm standard deviations $(n=3)$. (c) Time-dependent in vivo fluorescence images of AF647-labeled PEG40-110, PEG40-110-RDG2, and PEG40110-RGD2 particles intravenously injected into mice with U-87 MG xenograft tumors.

Surface modification of particles typically alters their surface chemistry and therefore influences their biological behavior. ${ }^{43,44}$ To investigate whether peptide modification 
influenced tumor targeting of the PEG particles in vivo, PEG40-110, PEG40-110-RDG2, and PEG40-110-RGD2 particles were labeled with AF647 and intravenously injected into tumorbearing nude mice $(n=3)$. The main organs and tumors were harvested to measure the fluorescence signal at $48 \mathrm{~h}$ after intravenous injection. As shown in Figure 4b, the PEG40-110RGD2 particles displayed significantly higher specific tumor accumulation and higher nonspecific liver and spleen accumulation compared with the PEG40-110-RDG2 particles, which in turn had higher tumor, liver, and spleen accumulation than the PEG40-110 particles. Interestingly, these results suggest that translation from in vitro static or flow experiments to in vivo experiments is not straightforward, as some of the results differ. Namely, both types of peptide-modified PEG particles had higher nonspecific accumulation than the nonfunctionalized PEG particles. The modification of PEG particles with RGD or RDG will change the surface chemistry of the PEG particles and therefore influence their interactions with immune cells (e.g., monocytes or macrophages), which may affect their biodistribution in liver and spleen. The fluorescence images of the mice demonstrated that the PEG40-110-RGD2

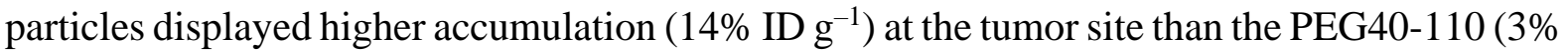
ID $\mathrm{g}^{-1}$ ) and PEG40-110-RDG2 (7\% ID $\mathrm{g}^{-1}$ ) particles (Figure 4c). To demonstrate the distribution of the PEG40-110-RGD2 particles at the tumor site, the tumor was sectioned and stained for imaging. As shown in Figure S6, the PEG40-110-RGD2 particles could accumulate in the tumor via blood circulation and extravasation and could be internalized by tumor cells, as consistent with the results from the MCS association experiments.

\section{CONCLUSIONS}


In summary, we engineered RGD-functionalized PEG particles using an MS templating method and investigated their biological behaviors. We specifically focused on optimizing the design and engineering process to balance the stealth and targeting properties of the particles. For the RGD-functionalized PEG particles, increasing the molecular weight of PEG decreased the targeting performance to U-87 MG cells, which could be balanced by increasing the RGD modification ratio. Compared with the nonfunctionalized PEG particles, the PEG-RGD particles effectively targeted cancer cells under both static and flow conditions, while the PEG and PEG-RDG particles maintained their stealth properties. RDG modification of the PEG particles increased nonspecific interactions and led to improved passive targeting in vivo. In contrast, RGD targeting allowed for higher tumor accumulation, while also increasing nonspecific liver and spleen accumulation. The RGD-integrin interaction occurs following delivery by blood circulation and extravasation, which facilitates the internalization of the PEG-RGD particles into $\alpha_{\mathrm{v}} \beta_{3}$ integrin-bearing U-87 MG tumor cells.

Our experiments show that moving from static cell culture to flow cell culture and spheroids gives consistent results for targeting and stealth. However, moving to the more complex biological environment of the circulatory system in vivo yields different results. For example, the stealth properties displayed by the PEG-RDG particles are similar to those displayed by the PEG particles in vitro, whereas the nonspecific accumulation is higher for the PEG-RDG particles than for the PEG particles in vivo. These discrepancies highlight challenges for successfully predicting translation from in vitro to in vivo systems. Overall, the reported targeted PEG particles represent a platform toward balancing nonspecific interactions with 
targeting-induced cell internalization, which is favorable for the study of bio-nano interactions and improved drug delivery performance.

\section{ASSOCIATED CONTENT}

Supporting Information. NMR spectra of peptide-functionalized 8-arm-PEG-NHS; molecular structure of 8-arm-PEG-NH 2 and scheme of the modification of 8-arm-PEG-NHS with cRGDfK or cRDGfK; CLSM images of U-87 MG cells incubated with targeted PEG particles; setup for microfluidic assay; CLSM images of a U-87 MG cell spheroid incubated with targeted PEG particles and the tumor region showing the distribution of targeted PEG particles; flow cytometry gating strategy; movies showing cell association of PEG40-1000RGD2 and PEG40-1000-RDG2 particles with U-87 MG cells under flow conditions; and the MIRIBEL checklist for reporting research in bio-nano science. The Supporting Information is available free of charge on the ACS Publications website.

\section{AUTHOR INFORMATION}

\section{Corresponding Author}

*E-mail: christoph.hagemeyer@monash.edu (C. E. H.)

*E-mail: fcaruso@unimelb.edu.au (F. C.)

\section{Author Contributions}

The manuscript was written through contributions of all authors. All authors have given approval to the final version of the manuscript. $\perp$ J. C., K. A., and Y. J. contributed equally to this work. 


\section{Notes}

The authors declare no conflict of interest.

\section{ACKNOWLEDGMENT}

This work was conducted and funded by the Australian Research Council Centre of Excellence in Convergent Bio-Nano Science and Technology (project number CE140100036) and the National Health and Medical Research Council (NHMRC) (grant no. 1098867 to K.A. and grant no. 1078118 to C.E.H.). F.C. acknowledges the award of an NHMRC Senior Principal Research Fellowship (GNT1135806). J.C. acknowledges the National Natural Science Foundation of China $(21603120,21872085)$. This work was performed in part at the Materials Characterisation and Fabrication Platform (MCFP) at The University of Melbourne and the Victorian Node of the Australian National Fabrication Facility (ANFF). We thank Mr. Zhiliang Gao at Shandong University for the NMR analysis.

\section{REFERENCES}

(1) Blanco, E.; Shen, H.; Ferrari, M. Principles of Nanoparticle Design for Overcoming Biological Barriers to Drug Delivery. Nat. Biotechnol. 2015, 33, 941-951.

(2) Liu, J.; Li, M.; Luo, Z.; Dai, L.; Guo, X.; Cai, K. Design of Nanocarriers Based on Complex Biological Barriers In Vivo for Tumor Therapy. Nano Today 2017, 15, 56-90.

(3) Cui, J.; Richardson, J. J.; Björnmalm, M.; Faria, M.; Caruso, F. Nanoengineered Templated Polymer Particles: Navigating the Biological Realm. Acc. Chem. Res. 2016, 49, $1139-1148$.

(4) Petros, R. A.; DeSimone, J. M. Strategies in the Design of Nanoparticles for Therapeutic 
Applications. Nat. Rev. Drug Discovery 2010, 9, 615-627.

(5) Suk, J. S.; Xu, Q.; Kim, N.; Hanes, J.; Ensign, L. M. PEGylation as a Strategy for Improving Nanoparticle-Based Drug and Gene Delivery. Adv. Drug Delivery Rev. 2016, 99, $28-51$.

(6) Yang, Q.; Jones, S. W.; Parker, C. L.; Zamboni, W. C.; Bear, J. E.; Lai, S. K. Evading Immune Cell Uptake and Clearance Requires PEG Grafting at Densities Substantially Exceeding the Minimum for Brush Conformation. Mol. Pharm. 2014, 11, 1250-1258.

(7) Perry, J. L.; Reuter, K. G.; Kai, M. P.; Herlihy, K. P.; Jones, S. W.; Luft, J. C.; Napier, M.; Bear, J. E.; DeSimone, J. M. PEGylated PRINT Nanoparticles: The Impact of PEG Density on Protein Binding, Macrophage Association, Biodistribution, and Pharmacokinetics. Nano Lett. 2012, 12, 5304-5310.

(8) Du, X. J.; Wang, J. L.; Liu, W. W.; Yang, J. X.; Sun, C. Y.; Sun, R.; Li, H. J.; Shen, S.; Luo, Y. L.; Ye, X. D.; Zhu, Y. H.; Yang, X. Z.; Wang, J. Regulating the Surface Poly(ethylene glycol) Density of Polymeric Nanoparticles and Evaluating Its Role in Drug Delivery In Vivo. Biomaterials 2015, 69, 1-11.

(9) Cui, J.; De Rose, R.; Alt, K.; Alcantara, S.; Paterson, B. M.; Liang, K.; Hu, M.; Richardson, J. J.; Yan, Y.; Jeffery, C. M.; Price, R. I.; Peter, K.; Hagemeyer, C. E.; Donnelly, P. S.; Kent, S. J.; Caruso, F. Engineering Poly(ethylene glycol) Particles for Improved Biodistribution. ACS Nano 2015, 9, 1571-1580.

(10) Cui, J.; Björnmalm, M.; Liang, K.; Xu, C.; Best, J. P.; Zhang, X.; Caruso, F. Super-Soft Hydrogel Particles with Tunable Elasticity in a Microfluidic Blood Capillary Model. Adv. Mater. 2014, 26, 7295-7299. 
(11) Yu, B.; Wei, H.; He, Q.; Ferreira, C. A.; Kutyreff, C. J.; Ni, D.; Rosenkrans, Z. T.; Cheng, L.; Yu, F.; Engle, J. W.; Lan, X.; Cai, W. Efficient Uptake of ${ }^{177}$ Lu-Porphyrin-PEG Nanocomplexes by Tumor Mitochondria for Multimodal-Imaging-Guided Combination Therapy. Angew. Chem. Int. Ed. 2018, 57, 218-222.

(12) Dai, Y.; Guo, J.; Wang, T. Y.; Ju, Y.; Mitchell, A. J.; Bonnard, T.; Cui, J.; Richardson, J. J.; Hagemeyer, C. E.; Alt, K.; Caruso, F. Self-Assembled Nanoparticles from Phenolic Derivatives for Cancer Therapy. Adv. Healthcare Mater. 2017, 6, 1700467.

(13) Dai, Y.; Yang, Z.; Cheng, S.; Wang, Z.; Zhang, R.; Zhu, G.; Wang, Z.; Yung, B. C.; Tian, R.; Jacobson, O.; Xu, C.; Ni, Q.; Song, J.; Sun, X.; Niu, G.; Chen, X. Toxic Reactive Oxygen Species Enhanced Synergistic Combination Therapy by Self-Assembled Metal-Phenolic Network Nanoparticles. Adv. Mater. 2018, 30, 1704877.

(14) Björnmalm, M.; Thurecht, K. J.; Michael, M.; Scott, A. M.; Caruso, F. Bridging BioNano Science and Cancer Nanomedicine. ACS Nano 2017, 11, 9594-9613.

(15) Gu, F.; Zhang, L.; Teply, B. A.; Mann, N.; Wang, A.; Radovic-Moreno, A. F.; Langer, R.; Farokhzad, O. C. Precise Engineering of Targeted Nanoparticles by Using Self-Assembled Biointegrated Block Copolymers. Proc. Natl. Acad. Sci. 2008, 105, 2586-2591.

(16) Chen, H.; Zhang, W.; Zhu, G.; Xie, J.; Chen, X. Rethinking Cancer Nanotheranostics. Nat. Rev. Mater. 2017, 2, 17024.

(17) Sun, Q.; Zhou, Z.; Qiu, N.; Shen, Y. Rational Design of Cancer Nanomedicine: Nanoproperty Integration and Synchronization. Adv. Mater. 2017, 29, 1606628.

(18) Fu, X.; Hosta-Rigau, L.; Chandrawati, R.; Cui, J. Multi-Stimuli-Responsive Polymer Particles, Films, and Hydrogels for Drug Delivery. Chem 2018, 4, 2084-2107. 
(19) Ju, Y.; Cui, J.; Sun, H.; Mullner, M.; Dai, Y.; Guo, J.; Bertleff-Zieschang, N.; Suma, T.; Richardson, J. J.; Caruso, F. Engineered Metal-Phenolic Capsules Show Tunable Targeted Delivery to Cancer Cells. Biomacromolecules 2016, 17, 2268-2276.

(20) Dai, Q.; Bertleff-Zieschang, N.; Braunger, J. A.; Bjornmalm, M.; Cortez-Jugo, C.; Caruso, F. Particle Targeting in Complex Biological Media. Adv. Healthcare Mater. 2018, 7, 1700575.

(21) Cui, J.; Björnmalm, M.; Ju, Y.; Caruso, F. Nanoengineering of Poly(ethylene glycol) Particles for Stealth and Targeting. Langmuir 2018, 34, 10817-10827.

(22) Cui, J.; Ju, Y.; Houston, Z. H.; Glass, J. J.; Fletcher, N. L.; Alcantara, S.; Dai, Q.; Howard, C. B.; Mahler, S. M.; Wheatley, A. K.; Rose, R. D.; Brannon, P. T.; Paterson, B. M.; Donnelly, P. S.; Thurecht, K. J.; Caruso, F.; Kent, S. J. Modulating Targeting of Poly(ethylene glycol) Particles to Tumor Cells Using Bispecific Antibodies. Adv. Healthcare Mater. 2019, 8, 1801607. (23) Kathleen, M. M.; Ananth, A.; Ravi, V. B. Decreased Circulation Time Offsets Increased Efficacy of PEGylated Nanocarriers Targeting Folate Receptors of Glioma. Nanotechnology 2007, 18, 385101 .

(24) Dai, Q.; Walkey, C.; Chan, W. C. Polyethylene Glycol Backfilling Mitigates the Negative Impact of the Protein Corona on Nanoparticle Cell Targeting. Angew. Chem. Int. Ed. 2014, 53, 5093-5096.

(25) Ma, K.; Mendoza, C.; Hanson, M.; Werner-Zwanziger, U.; Zwanziger, J.; Wiesner, U. Control of Ultrasmall Sub-10 nm Ligand-Functionalized Fluorescent Core-Shell Silica Nanoparticle Growth in Water. Chem. Mater. 2015, 27, 4119-4133.

(26) Miura, Y.; Takenaka, T.; Toh, K.; Wu, S.; Nishihara, H.; Kano, M. R.; Ino, Y.; Nomoto, 
T.; Matsumoto, Y.; Koyama, H.; Cabral, H.; Nishiyama, N.; Kataoka, K. Cyclic RGD-Linked Polymeric Micelles for Targeted Delivery of Platinum Anticancer Drugs to Glioblastoma through the Blood-Brain Tumor Barrier. ACS Nano 2013, 7, 8583-8592.

(27) Chen, Q.; Wang, X.; Wang, C.; Feng, L.; Li, Y.; Liu, Z. Drug-Induced Self-Assembly of Modified Albumins as Nano-Theranostics for Tumor-Targeted Combination Therapy. ACS Nano 2015, 9, 5223-5233.

(28) Chen, H.; Niu, G.; Wu, H.; Chen, X. Clinical Application of Radiolabeled RGD Peptides for PET Imaging of Integrin $\alpha_{v} \beta_{3}$. Theranostics 2016, 6, 78-92.

(29) Phillips, E.; Penate-Medina, O.; Zanzonico, P. B.; Carvajal, R. D.; Mohan, P.; Ye, Y.; Humm, J.; Gönen, M.; Kalaigian, H.; Schöder, H.; Strauss, H. W.; Larson, S. M.; Wiesner, U.; Bradbury, M. S. Clinical Translation of an Ultrasmall Inorganic Optical-PET Imaging Nanoparticle Probe. Sci. Transl. Med. 2014, 6, 260ra149.

(30) Chen, F.; Ma, K.; Benezra, M.; Zhang, L.; Cheal, S. M.; Phillips, E.; Yoo, B.; Pauliah, M.; Overholtzer, M.; Zanzonico, P.; Sequeira, S.; Gonen, M.; Quinn, T.; Wiesner, U.; Bradbury, M. S. Cancer-Targeting Ultrasmall Silica Nanoparticles for Clinical Translation: Physicochemical Structure and Biological Property Correlations. Chem. Mater. 2017, 29, 8766-8779.

(31) Kawamura, W.; Miura, Y.; Kokuryo, D.; Toh, K.; Yamada, N.; Nomoto, T.; Matsumoto, Y.; Sueyoshi, D.; Liu, X.; Aoki, I.; Kano, M. R.; Nishiyama, N.; Saga, T.; Kishimura, A.; Kataoka, K. Density-Tunable Conjugation of Cyclic RGD Ligands with Polyion Complex Vesicles for the Neovascular Imaging of Orthotopic Glioblastomas. Sci. Technol. Adv. Mater. 2015, 16, 035004. 
(32) Faria, M.; Björnmalm, M.; Thurecht, K. J.; Kent, S. J.; Parton, R. G.; Kavallaris, M.; Johnston, A. P. R.; Gooding, J. J.; Corrie, S. R.; Boyd, B. J.; Thordarson, P.; Whittaker, A. K.; Stevens, M. M.; Prestidge, C. A.; Porter, C. J. H.; Parak, W. J.; Davis, T. P.; Crampin, E. J.; Caruso, F. Minimum Information Reporting in Bio-Nano Experimental Literature. Nat. Nanotechnol. 2018, 13, 777-785.

(33) Wang, J.-G.; Zhou, H.-J.; Sun, P.-C.; Ding, D.-T.; Chen, T.-H. Hollow Carved SingleCrystal Mesoporous Silica Templated by Mesomorphous Polyelectrolyte-Surfactant Complexes. Chem. Mater. 2010, 22, 3829-3831.

(34) Zhang, K.; Xu, L. L.; Jiang, J. G.; Calin, N.; Lam, K. F.; Zhang, S. J.; Wu, H. H.; Wu, G. D.; Albela, B.; Bonneviot, L.; Wu, P. Facile Large-Scale Synthesis of Monodisperse Mesoporous Silica Nanospheres with Tunable Pore Structure. J. Am. Chem. Soc. 2013, 135, $2427-2430$.

(35) Björnmalm, M.; Cui, J.; Bertleff-Zieschang, N.; Song, D.; Faria, M.; Rahim, M. A.; Caruso, F. Nanoengineering Particles through Template Assembly. Chem. Mater. 2017, 29, 289-306.

(36) Lee, M. H.; Kim, J. Y.; Han, J. H.; Bhuniya, S.; Sessler, J. L.; Kang, C.; Kim, J. S. Direct Fluorescence Monitoring of the Delivery and Cellular Uptake of a Cancer-Targeted RGD Peptide-Appended Naphthalimide Theragnostic Prodrug. J. Am. Chem. Soc. 2012, 134, $12668-$ 12674.

(37) Farokhzad, O. C.; Khademhosseini, A.; Jon, S.; Hermmann, A.; Cheng, J.; Chin, C.; Kiselyuk, A.; Teply, B.; Eng, G.; Langer, R. Microfluidic System for Studying the Interaction of Nanoparticles and Microparticles with Cells. Anal. Chem. 2005, 77, 5453-5459. 
(38) Broda, E.; Mickler, F. M.; Lachelt, U.; Morys, S.; Wagner, E.; Brauchle, C. Assessing Potential Peptide Targeting Ligands by Quantification of Cellular Adhesion of Model Nanoparticles Under Flow Conditions. J. Controlled Release 2015, 213, 79-85.

(39) Albanese, A.; Lam, A. K.; Sykes, E. A.; Rocheleau, J. V.; Chan, W. C. W. Tumour-on-aChip Provides an Optical Window into Nanoparticle Tissue Transport. Nat. Commun. 2013, 4, 2718.

(40) Björnmalm, M.; Yan, Y.; Caruso, F. Engineering and Evaluating Drug Delivery Particles in Microfluidic Devices. J. Controlled Release 2014, 190, 139-149.

(41) Korin, N.; Kanapathipillai, M.; Matthews, B. D.; Crescente, M.; Brill, A.; Mammoto, T.; Ghosh, K.; Jurek, S.; Bencherif, S. A.; Bhatta, D.; Coskun, A. U.; Feldman, C. L.; Wagner, D. D.; Ingber, D. E. Shear-Activated Nanotherapeutics for Drug Targeting to Obstructed Blood Vessels. Science 2012, 337, 738-742.

(42) Friedrich, J.; Seidel, C.; Ebner, R.; Kunz-Schughart, L. A. Spheroid-Based Drug Screen: Considerations and Practical Approach. Nat. Protoc. 2009, 4, 309.

(43) Qhattal, H. S. S.; Hye, T.; Alali, A.; Liu, X. Hyaluronan Polymer Length, Grafting Density, and Surface Poly(ethylene glycol) Coating Influence In Vivo Circulation and Tumor Targeting of Hyaluronan-Grafted Liposomes. ACS Nano 2014, 8, 5423-5440.

(44) Song, D.; Cui, J.; Sun, H.; Nguyen, T. H.; Alcantara, S.; De Rose, R.; Kent, S. J.; Porter, C. J. H.; Caruso, F. Templated Polymer Replica Nanoparticles to Facilitate Assessment of Material-Dependent Pharmacokinetics and Biodistribution. ACS Appl. Mater. Interfaces 2017, 9, 33683-33694. 
TABLE OF CONTENTS

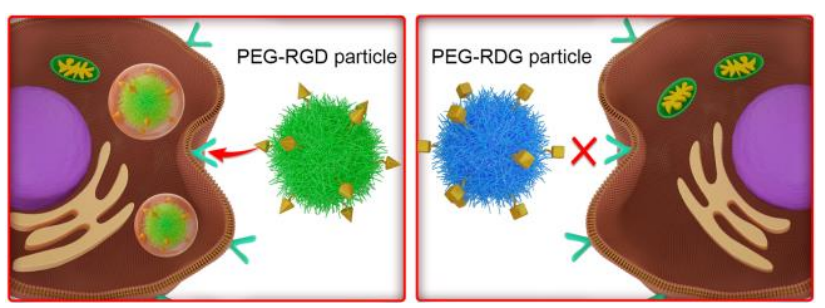

